\title{
Command Acknowledge through Tactile Feedback Improves the Usability of an EMG-based Interface for the Frontalis Muscle
}

This is the peer reviewed version of the following article:

Original:

Franco, L., Salvietti, G., Prattichizzo, D. (2019). Command Acknowledge through Tactile Feedback Improves the Usability of an EMG-based Interface for the Frontalis Muscle. In Proc. 2019 IEEE World Haptics Conference (WHC) (pp.574-579). IEEE [10.1109/WHC.2019.8816133].

Availability:

This version is availablehttp://hdl.handle.net/11365/1082333

since 2020-07-01T12:06:11Z

Publisher:

IEEE

Published:

DOI:10.1109/WHC.2019.8816133

Terms of use:

Open Access

The terms and conditions for the reuse of this version of the manuscript are specified in the publishing policy. Works made available under a Creative Commons license can be used according to the terms and conditions of said license.

For all terms of use and more information see the publisher's website.

(Article begins on next page) 


\title{
Command Acknowledge through Tactile Feedback Improves the Usability of an EMG-based Interface for the Frontalis Muscle
}

\author{
Leonardo Franco ${ }^{1,2}$, Gionata Salvietti ${ }^{1,2}$ and Domenico Prattichizzo ${ }^{1,2}$
}

\begin{abstract}
This work presents a study on the effectiveness of tactile feedback for the acknowledgement of a correct command detection in an EMG-based interface for the frontalis muscle. EMG interfaces are increasingly used in assistive robotics to control robots exploiting the repeatability and robustness of the electromyographic signal. However, in many application a feedback about the correct detection of an input is often missed and the user has to wait for the device motion in order to understand if his/her will has been correctly detected by the system. We demonstrate with a user study involving fifteen subjects, that a simple vibrotactile feedback can reduce the muscular effort and the time needed to execute a sequence of action commanded by an EMG device. As a case study, an EMG interface for the frontalis muscle has been used, however proposed results could be extended to EMG interfaces designed for other muscles, e.g., for prosthesis or exoskeleton control.
\end{abstract}

\section{INTRODUCTION}

Assistive robotics is gaining an increasing importance due to novel technological and scientific progresses. Alongside novel and sophisticated robots that can assist physically impaired subjects, several solutions for Human-Machine (HMI) and Human-Computer Interfaces (HCI) have been developed. Such interfaces usually exploit bio-signals that can be voluntary controlled by the user. Muscular contraction measured through electromyographical (EMG) activity of skeletal muscles is a classic example of bio-signal used for interfaces. The technology necessary to process such biosignal is becoming smaller and more powerful. This allows to develop wearable interfaces which interact with the human body.

Among the body muscles, the frontalis muscle has been selected by several research groups to build a HMI or a $\mathrm{HCI}$, for example to realize a computer pointing system [1] [2] [3], to recognize facial gestures [4] [5], or to move a wheelchair [6]. One of the main reason for choosing this muscle is that it is always spared in case of a motor stroke either of the left or of the right hemisphere due to its bilateral cortical representation and it is usually usable by tetraplegic patients.

Our research group developed an EMG interface called eCap for the control of an assistive device called the Robotic Sixth Finger. This assistive device is a supernumerary robotic finger that allows hemiparetic chronic stroke patients to compensate for the missed grasping capability and to perform

The research leading to these results has received funding from the European Union's Horizon 2020 Research and Innovation Programme under Grant Agreement 688857 of the project "SoftPro".

${ }^{1}$ Dept. of Information Engineering and Mathematics, Univ. of Siena, Italy. (e-mail: [franco, salviettigio, prattichizzo]@diism.unisi.it).

${ }^{2}$ Dept. of Advanced Robotics, Istituto Italiano di Tecnologia, Italy. again a set of bimanual tasks typical of activities of daily living [7], [8]. The robotic extra finger can be worn at the patient paretic forearm and can restrain the motion of a desired object by wrapping around the object and pushing it toward the paretic arm realising a hybrid human-robot grasp. The eCap was a wearable wireless EMG interface where electrodes, acquisition and signal conditioning boards were embedded in a cap. For chronic patients it is generally difficult to generate repeatable EMG patterns in their paretic upper limb due to the weakness in muscle contraction control. For this reason, we coupled the flexion/extension motion of the robotic device with the contraction of the frontalis muscle. The activity of the muscle was recognized filtering the raw EMG signal and setting a threshold as a percentage of the maximum voluntary contraction of the muscle. The user could contract this muscle by moving the eyebrows upwards. The movement of the robotic device was then controlled by using a Finite State Machine (FSM) whose states were spanned through a trigger signal obtained from the eCap interface. The outputs of the FSM were predefined commands based on sequences of input signals. We considered a finite number of states, transition between those states, and commands. States represented predefined motion commands for the robotic device and transition actions were associated with contractions of the frontalis muscle. The FSM was necessary to keep as simple as possible and low cognitively demanding the muscular activity requested to the patients to trigger a certain event. We successfully started using this interface in pilot studies involving chronic stroke patients. However, we quickly recognize the need for a feedback to warn the user about the current FSM status and to inform about the correct detection of a command. Our first solution was a visual feedback consisting in a LEDs board which represented different states, see in [9] [8]. However, this kind of feedback resulted cognitively demanding because, while the user was moving the affected arm to center the object to grasp in the workspace of the robotic finger, he/she had also to check the LEDs to be sure that the eCap received the correct input. Furthermore, such a visual feedback suffers from possible visual obstruction in case the user wears something that could cover the LEDs.

In this work, the visual feedback has been substituted with a tactile feedback, implemented by a vibromotor which gives cues on the head. This solution does not overload the user with additional visual informations and is more intimate, because the user is the only person that can notice the feedback. The aim of this study is to demonstrate that a tactile feedback of the correct detection of a command can improve 
the usability of an EMG interface. The haptic feedback has been usually used in literature when the EMG interfaces have been used to control prostheses [10] [11] [12] [13] or exoskeletons [14] [15]. The feedback is thus contributing to gather information about the interaction of the device with the environment. To the best of our knowledge, little studies exists on the importance of command acknowledge through tactile feedback. We used the haptic feedback to inform the user about the correct detection of a control input to the system. We proved that the acknowledge of the input is very useful to the user since it reduces the cognitive delay between the intended action, the muscle movement and the actual robot action. We demonstrated that haptic feedback improve the usability of the interface and help in exploiting the functionalities of the Robotic Sixth Finger through a user study involving fifteen subjects.

The rest of the paper is organised as follows. In Section II, a detailed description of the EMG interface is provided. In Section III, we describe the experimental setup used to evaluate the tactile feedback, whereas the obtained results are reported in Section IV. In Section V, a discussion on the proposed results is reported, whereas in Section VI conclusion and future work are outlined.

\section{The FRONTALis MUSCLE INTERFACE}

In this section, we describe in detail the frontalis muscle interface. The device consist of a headband that provides surface electromyographic (EMG) signal acquisition, on-board data processing, Bluetooth connection, and haptic/vibration feedback, see Fig. 1.

The headband is made out of an elastic band with sewed velcro strips to adapt the band length to the user head size. Non-gelled electrodes are used since they result more easy to wear and to be adjusted on the user front to improve the comfort. Non-gelled electrodes result also less expensive on a long term used since they have not to be replaced after few time of usage. The housing for surface EMG electrodes is $3 \mathrm{D}$ printed with two lateral belt loop so that is easy to tighten and lock the elastic band.

The electrode housing is a critical aspect of the device in terms of mechanical coupling since it has to correctly place the electrode surfaces over the frontalis muscle getting rid of the different head curvature among different users. The same part has to retain comfort for prolonged use, but at the same time, it has to exert the correct pressure against the skin to guarantee enough stability to the skin-electrode interface since the electrodes used are not gelled. To address these issues, the curved rigid 3D printed part tied to the elastic band has been designed with embedding a compliant housing for the electrodes. A set of springs push a 3D printed flexible socket for the electrodes allowing the electrode plane both to tilt, aligning with the head surface, and provide pressure to the electrodes. Details of the obtained electrodes housing are reported in Fig. 2. Three reusable non-gelled electrodes coated with $\mathrm{Ag} / \mathrm{AgCl}$ polymer $10 \mathrm{~mm}$ in diameter and $2 \mathrm{~mm}$ thick have been used. The EMG signal is amplified with an instrumentation amplifier and passes through an analogic filter both embedded in a compact board. Both electrodes and amplifier (BITalino, Portugal) are designed for EMG acquisition, with a gain of 1009 , input range of $\pm 1.65 \mathrm{mV}$, filter bandwidth $25 \div 482 \mathrm{~Hz}$, common mode rejection ratio of $80 \mathrm{~dB}$, input impedance of $10 \mathrm{G} \Omega$.

The haptic feedback is realised through a vibrotactile motor. A rigid 3D printed case containing an Eccentric Rotating Mass (ERM) motor (Precision MicroDrives, United Kingdom) is fixed to the elestic band in the occipital position, see right hand side of Fig. 1 and the bottom part of Fig. 2. Two main reasons motivate this decision for the vibromotor positioning. Firstly, Jesus Oliveira et al. proved in [16], that the ear mid-line area has to be avoided for placing vibration cues since vibration can result in undesirable disturbing sounds for the user. Secondly, the positioning in the head frontal region cannot be considered since the front is occupied by the acquisition stage and vibrations could affect the EMG recording during operation.

A Lithium-Polymer battery was used to power the system since all circuitry are supplied with $3.3 \mathrm{~V}$. It was secured to the headband by sewing a pocket directly on the elastic headband, see the center of Fig. 1. A switch button is used to interrupt battery connection.

The sampling and data processing of the EMG signal is performed with the Teensy 3.2 (PJRC, USA), a cheap yet powerful micro-controller which mounts a 32-bit ARM Cortex-M4 module. It can sample signals up to 16-bit resolution, and it is over-clockable up to $96 \mathrm{MHz}$, allowing fast/real-time data processing.

The headband is interfaced with the Sixth Finger through Bluetooth 2.1, implemented in the RN-42 module of Roving Networks.

A switch was added to the headband to swap between a calibration mode and an operation mode.

A custom printed circuit board was realized to connect all the electronics hereupon described, avoiding most of the messy connections and providing the hardware with reliability and robustness.

\section{EXPERIMENTAL SETUP}

In this section, we describe the experimental setup used to test the effect of haptic feedback on the use of the EMG interface. We selected a possible task to be executed with the help of the interface that consists on controlling the flexion/extension of the Robotic Sixth Finger. We already proved in [9], [8], [7] the importance of an EMG interface for controlling grasp assistive tools. Readers are referred to those references also for further details on the Robotic Sixth Finger functioning and realization.

The robotic finger and the interface are connected through Bluetooth antennas. An additional antenna connects the Sixth Finger to a laptop to gather processed data and to visualize the raw EMG signal during the calibration operation, see Fig. 3. The real-time signal processing runs at the rate of 1 $\mathrm{kHz}$ as well as the signal sampling rate. At each new sample, the raw EMG signal processing consists of the computation of the zero mean signal, subtracting the mean of a sufficiently 

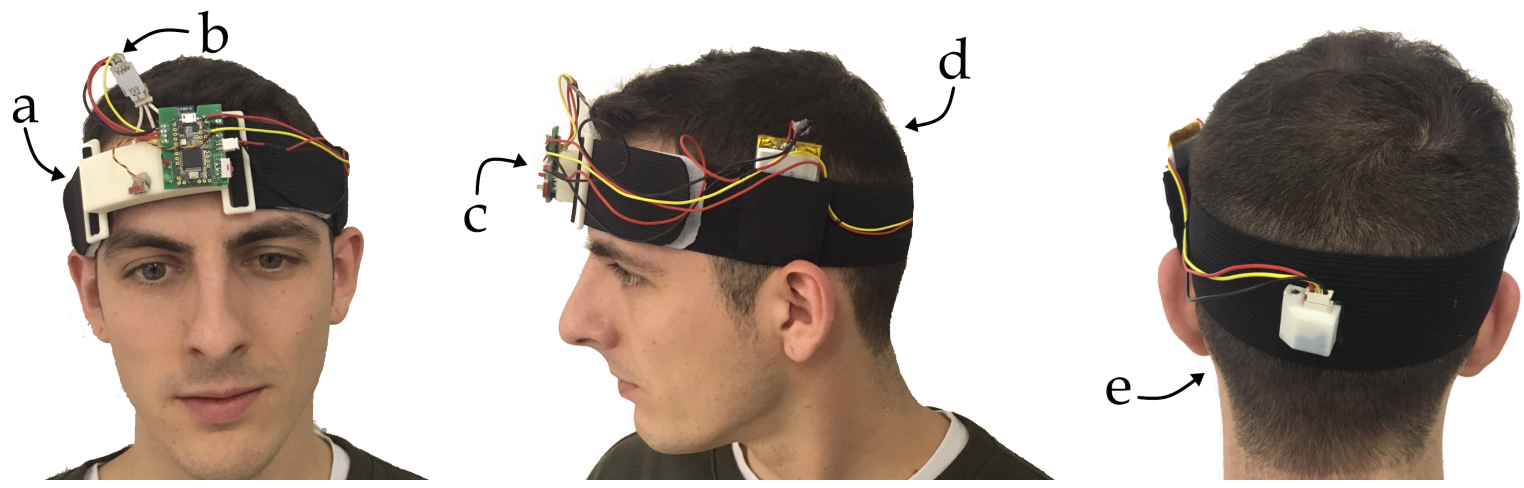

Fig. 1. The Frontalis muscle interface front, side and back view. Arrows indicate: (a), 3D printed electrodes socket with loops for elastic band; (b), EMG conditioning board; (c), sampling and data processing board with Bluetooth module mounted on a custom PCB; (d), Li-Po battery; (e), vibration motor (ERM) for the haptic feedback, embedded in a 3D printed socket.

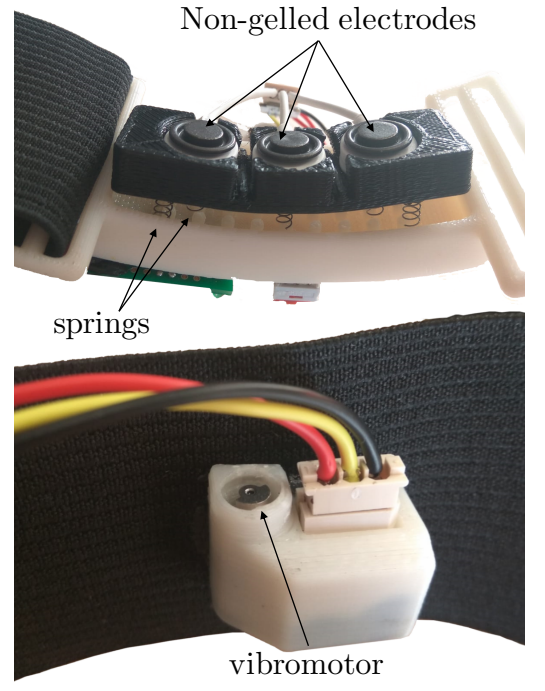

Fig. 2. Details of 3D printed parts of the frontalis muscle interface. Top part shows EMG electrodes housing with compliant connection through springs to the rigid case made of ABS material. The black part where electrodes are fixed is 3D printed with flexible thermoplastic polyurethane. On the bottom part, the vibromotor case made of ABS that also embeds a current amplifier.

long buffer of the raw signal to the new incoming sample. It was empirically determined that 350 samples long buffer, was enough to ensure a stable estimate of the raw signal mean value.

Then, the Teager-Kaiser operator [17], a non-linear energy operator is applied to the last 3 samples, enhancing the signal to noise ratio. This is a discrete operator defined in time domain as

$$
\Psi_{d}[x(n)]=x^{2}(n)-x(n+1) x(n-1)
$$

and has been proven to enhance performances of data processing algorithm when dealing with EMG onset identification [18], [19]. A buffer of 350 samples of the energy of the signal is stored and the envelope of the signal is obtained by weighting the resulting 350 samples with a Gaussian window of weights $w_{G}[n]$ and summing them up together.
The Gaussian weights were defined as

$$
w_{G}[n]=\exp \left(\frac{-1}{2}\left(\frac{\alpha n}{\frac{N-1}{2}}\right)^{2}\right)
$$

with $-\frac{N-1}{2} \leq n \leq \frac{N-1}{2}, \alpha=2.5$ and $N=350$.

The obtained envelope is the convolution between the energy of the signal and a window of Gaussian weights, thus can be synthetically described as a modified moving average of the energy of the EMG signal. The envelope value $E_{E M G}$ at the time instant $t_{0}$ is obtained as

$$
E_{E M G}\left(t_{0}\right)=\sum_{n=0}^{N}\left(\Psi_{d}[x(n)]\right)^{2} w_{G}\left(n+\frac{N-1}{2}\right) .
$$

This envelope represents the instantaneous power exerted by the muscle and will be taken as an index of performance as reported in Section IV.

A calibration procedure is run for each subject before starting to execute the task. The calibration is needed to select the correct threshold for acknowledging an intentional contraction of the frontalis muscle. We used the Maximum Voluntary Contraction (MVC) technique for the threshold computation [20], [21]. The system records the highest value of the envelope during a single upright movement of the eyebrows in which the users slowly start increasing the contraction of the forehead muscle to reach their maximum effort. The single-threshold value defined is then defined as the $50 \%$ of the MVC, a level that is repeatable and sustainable for the subject without producing noticeable fatigue during the use of the device. This procedure limits the problems related to the high influence of detection condition on EMG signal amplitude that can change between electrode sites, subjects and even day to day measures of the same muscle site. During the calibration phase, the raw signal and the computed envelope are streamed via Bluetooth to the laptop. An operator checks whether the signal is correctly measured and not affected by artefacts due to electrodes' sliding over the skin of the user. After the calibration procedure, when the EMG envelope amplitude overcomes the threshold a counter verifies that the contraction persists in time for at 
least $50 \mathrm{~ms}$. This second threshold ensures that noise or short involuntary contraction are not assumed as a voluntary muscular activation.

Once a voluntary contraction is recognized by the device, the rising and falling edges of the muscle activation, are sent to the Sixth Finger through the Bluetooth connection. The microcontroller of the Sixth Finger receives the rising and falling edges and counts how much time elapses between them. A single activation is considered when a rising and a falling edge are received within a predefined time window. If two rising edges and falling edges are received inside the time window, then a double activation is identified. This is used to switch among states in the Finite State Machine (FSM) that runs in the the Sixth Finger microntroller. A detailed description of the FSM running on the Robotic Sixth Finger microcontroller can be found in [9]. In this work, we used a simplified version that is briefly summarized in the following. According to the current state of the robotic finger, the state of the FSM can change to

- "finger flexion" when a single contraction is detected, only if the finger is already in the completely open state;

- "finger extension" when two contractions are detected, only if the finger is already in the completely closed state;

- "null" the input is ignored.

In other words, if the eyebrows movement is performed one time in the time window the finger start to flex, if the eyebrows are moved upwards twice, the finger start to extend.

The task to perform is to open and close for two consequently times the robotic finger by moving upward the eyebrows, for a total of four commands to be interpreted by the interface. Two feedback conditions were considered:

- Haptic feedback (CF): A vibration burst acknowledges for the contraction detected contraction of the muscle. If there is a single muscle activation, the haptic feedback consists of a single vibration burst. If a second contraction is detected inside the given time window, a second and longer vibration is provided to the user. In other words, a single vibration burst correspond to the detection of the command "close the finger", whereas a vibration burst followed by a longer vibration correspond to the detection of the command "open the finger".

- No haptic feedback considered (N).

The vibration are generated by controlling the vibrotactile motor at full intensity for $100 \mathrm{~ms}$ in the case of a short burst, and for $300 \mathrm{~ms}$ in the case of the longer vibration. This is useful for the user since functionally differentiated pulse quality allows the user to clearly understand whether they managed to perform two movements in a row or failed in this task. Moreover, this differentiation in pulse length increases the confidence of the user in operating the robotic finger, because with this kind of feedback it is acknowledged that the system recognized the input. The acknowledge is faster than the actual movement of the robotic finger, in this way the user does not have to wait for it.

Three different lengths for the time windows were randomly

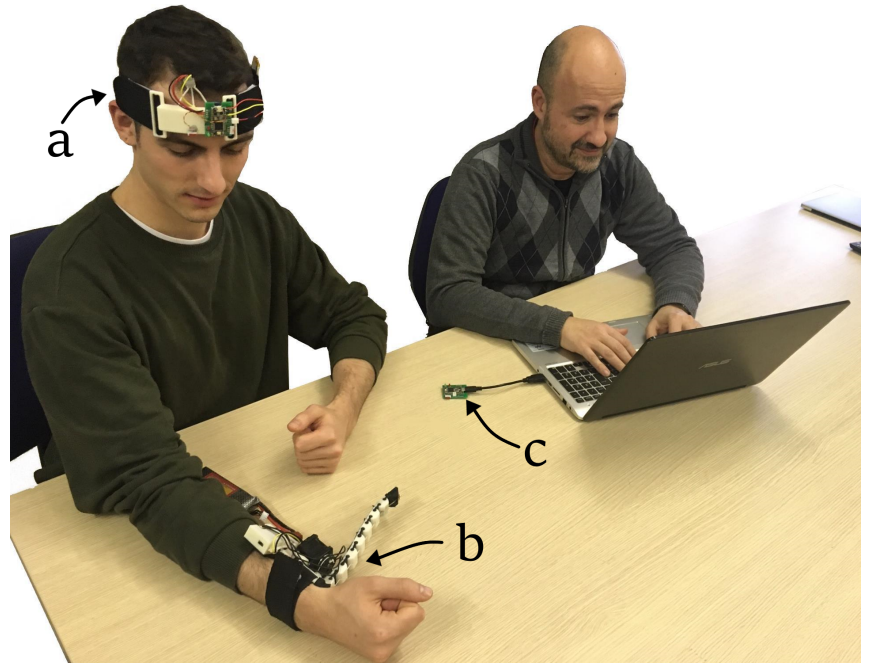

Fig. 3. Experimental setup. The user wears both the frontalis muscle interface (a) and the Robotic Sixth Finger (b). Data is received through a Bluetooth receiver (c)

used during the experiments so to limit possible learning effect. In fact, once the user familiarizes with the control interface, learns how much time available there is to complete the movements. The possible time window lengths were 800 , 1000 and $1200 \mathrm{~ms}$.

\section{RESUlTS}

Fifteen subjects ( 8 females, average age $28.5 \pm 6.8$ years) participated to the experiment. Seven of them had previous experience with haptic interfaces. None of the participants reported any deficiencies in their perception abilities and they were all naive as to the purpose of the study.

Each user repeated an open/close command for all combinations two times, with two feedback conditions, and with three possible time window lengths. This led to twelve consecutive open/close per experiment. The experiment is repeated twice gathering a total of twenty-four open/close commands per subject. We define as a task the sequence of two open/close commands. We considered two quantitative indexes to measure the role of tactile feedback for the EMG interface: we defined a Muscle Effort Index (MEI) to quantify the effort needed to accomplish a task with or without haptic feedback and, we considered the time need to accomplish a task with and without haptic feedback. In addition to the quantitative evaluation, we also measured users' experience through a questionnaire.

\section{A. Muscle effort index}

The goal of this evaluation is to determine whether the use of tactile feedback can reduce the muscular effort to execute a sequence of commands. To this aim, we defined the MEI as the measure of the total effort needed by each subject to accomplish the open/close task. The MEI is computed as follows. Every second we acquire 1000 EMG samples @ $1 \mathrm{kHz}$. For each sample it is possible to compute the envelope contributions as defined in Eq. (3). Summing all the 


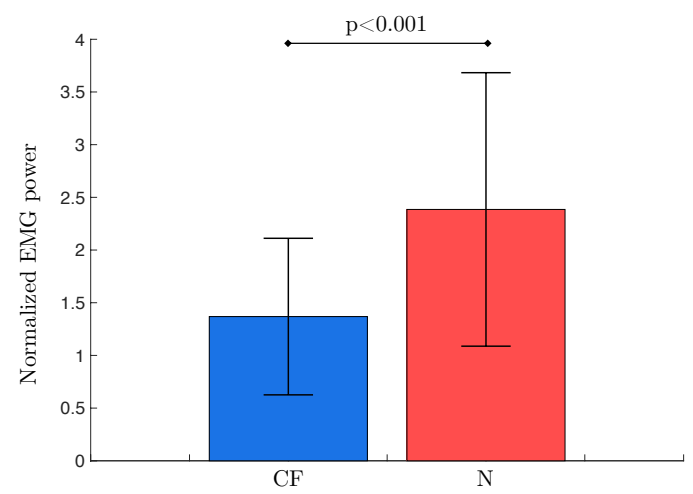

Fig. 4. Normalized MEI. Mean and standard deviation are plotted for the two considered conditions.

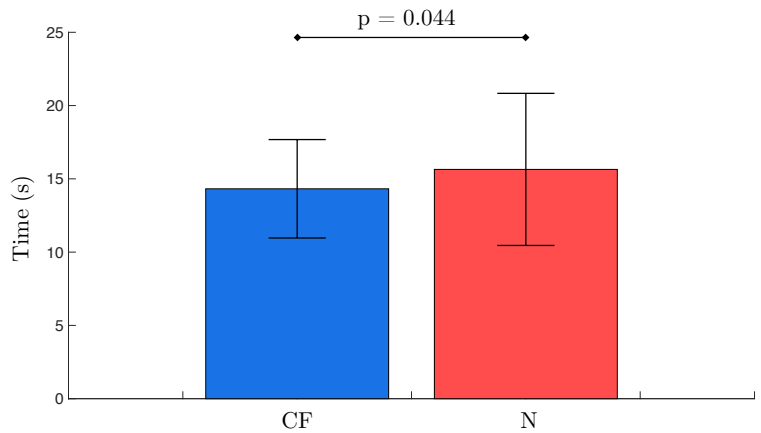

Fig. 5. Completion time. Means and standard deviations are plotted for the two considered conditions.

thousand sample contributions we obtain an estimation of the EMG power for the considered period of one second. If we normalize according to the highest EMG power measured, we obtain a series EMG power estimation ranging from 0 to 1 . This normalization is necessary since different muscle sizes and electrode positioning may result in different maximum values when computing the task total effort. We define the MEI for a task as the sum of all the normalized EMG power. These values represent the total effort to complete a single task. Fig. 4 shows mean and standard deviation of the MEI for each feedback condition. A paired T-test analysis revealed statistically significant difference between the tactile feedback and no feedback condition $(p<0.001)$.

\section{B. Completion time}

The goal of this evaluation is to verify whether the use tactile feedback can reduce the time necessary to execute a series of commands. Fig. 5 reports the mean and standard deviation of time values obtained by taking into account how many seconds elapsed between the first detected muscle contraction and the detection of a opening command, i.e. two muscle contraction in the time window. For each task this operation is repeated twice thus the reported times are the sum of the two open/close commands. A paired T-test analysis revealed statistically significant difference between the tactile feedback and no feedback condition $(p=0.044)$.

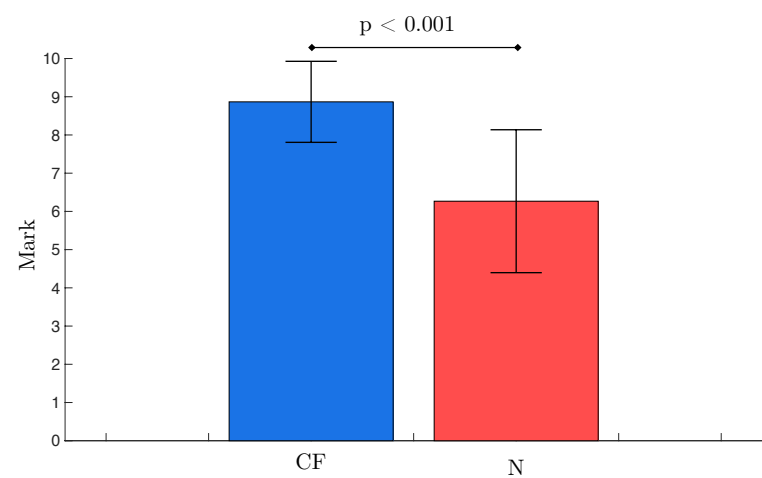

Fig. 6. Perceived effectiveness. Means and standard deviations of the marks are plotted for the two considered conditions.

\section{Perceived effectiveness}

At the end of the experiment, subjects were asked to rate, on a slider going from 0 to 10 , the effectiveness of each feedback condition in completing the given task. Fig. A paired T-test analysis revealed statistically significant difference between the marks assigned to tactile feedback and to no feedback condition $(p<0.001)$.

\section{DisCUSSION}

As we mentioned in the introduction, this work had been inspired by the real need of providing a non visual feedback to the patients using the Robotic Sixth Finger controlled through an EMG-based interface for the frontalis muscle. During the pilot studies performed, most of the patients asked for a possible acknowledge of the correct detection of a command gave by moving the eyebrows upward. The introduction of tactile feedback directly on the head of the user seems to be a promising way to solve this request. Our hypothesis was that the MEI value is expected to be higher when no haptic feedback is provided because in this case, the user has to wait for the movement of the robotic finger, that occurs at the end of the decision time window. In the elapsed time, usually the user continues to keep the eyebrows up when is no longer necessary. Warning the user with the described haptic cues before the finger action and immediately after the activation recognition, reduces the muscular effort needed to operate the robotic finger, diminishes the fatigue risk related to the use of the interface for a prolonged time and helps to reduce errors during the robotic finger control. Moreover, the presence of haptic feedback enhances the awareness of the user in using the system so that it becomes faster to execute the task of opening and closing the robotic finger.

We noticed that when no feedback is provided, is not easy to understand if the finger is actually receiving the opening trigger. This often leads users to try to repeat the eyebrow movement three or more times, losing trust in the interface or in their own ability to use it. Indeed, these ambiguities are strongly reduced when tactile feedback is given, since all the user were able to understand whether the interface recognized the double movement or not. 
Some participants reported that the experience with the interface was uncomfortable after the whole experiment, finding the cause of that both in the excessive pressure that electrodes exerted on the forehead and the repetition of the same task that involves a muscle that is not typically so often used voluntarily. Another issue to tackle in a future version of the device is the electrodes positioning. The frontalis muscle is not centred in the forehead, but is composed of two symmetrical muscles placed over the eyebrows. To have repeatability of EMG recording the mechanical coupling could be revisited, implementing an active adaptation to head curvature. Moreover, a second EMG channel could be added, monitoring the EMG activity of both frontalis muscles. Different actuators could be used to provide tactile cues, such as linear resonant actuator or piezoelectric elements.

Finally, in this work we only validated the effectiveness of the interface itself, but we did not consider that typical applications of EMG interfaces for assistive robotics may also require a feedback from the interaction of the device with the environment. This open interesting questions on the possible location of the device/devices providing these two distinguished feedbacks (acknowledge and interaction feedback). As reported by the users in the questionnaire, the haptic acknowledgement here studied is an element that enhance the usability of the device. This could be true not only for this interface/system but also in other prosthetic devices/orthosis which rely upon EMG inputs and haptic feedbacks. We point out that the different quality of the acknowledgement would not influence the force feedback, since is perceived as less natural by the user, yet very useful. With this in mind, we are willing to investigate whether that input acknowledgement haptic feedback could be implemented using the same actuators implied in the force feedback without any detrimental effect on it.

\section{CONCLUSION}

In this work, we analysed the contribution of tactile feedback on a EMG interface for the frontalis muscle. In particular, we focused on the evaluation of the importance of the acknowledge of the correct detection of a command through vibrations on the occipital side of the head. We demonstrated that a simple vibrotactile feedback can reduce the time to execute a series of commands. Moreover, also the muscular effort required to execute the series of commands is significantly reduced by the haptic feedback. All the fifteen subjects participating to the experiment indicate the tactile feedback as important add on for the EMG interface. We are currently testing the tactile feedback also with patients using the Robotic Sixth Finger. As a future work, we would like to test the vibrotactile command acknowledge also for different EMG interfaces, including EMG interfaces for prosthesis and exoskeleton.

\section{REFERENCES}

[1] A. Barreto, S. Scargle, and M. Adjouadi, "A practical emg-based human-computer interface for users with motor disabilities," 2000.
[2] N. M. Lopez, E. Orosco, E. Perez, S. Bajinay, R. Zanetti, M. E. Valentinuzzi, and G. de Tecnología Médica, "Hybrid human-machine interface to mouse control for severely disabled people," system, vol. 4, no. $11,2015$.

[3] M. J. Cler and C. E. Stepp, "Discrete vs. continuous mapping of facial electromyography for human-machine-interface control: Performance and training effects," IEEE Transactions on Neural Systems and Rehabilitation Engineering, vol. 23, no. 4, pp. 572-580, 2015.

[4] M. Hamedi, S.-H. Salleh, T. T. Swee, et al., "Surface electromyographybased facial expression recognition in bi-polar configuration," Journal of Computer Science, vol. 7, no. 9, pp. 1407-1415, 2011.

[5] M. Hamedi, S.-H. Salleh, M. Astaraki, and A. M. Noor, "Emg-based facial gesture recognition through versatile elliptic basis function neural network," Biomedical engineering online, vol. 12, no. 1, p. 73, 2013.

[6] L. Wei and H. Hu, "Emg and visual based hmi for hands-free control of an intelligent wheelchair," in Intelligent Control and Automation (WCICA), 2010 8th World Congress on, pp. 1027-1032, IEEE, 2010.

[7] G. Salvietti, I. Hussain, D. Cioncoloni, S. Taddei, S. Rossi, and D. Prattichizzo, "Compensating hand function in chronic stroke patients through the robotic sixth finger," Transaction on Neural System and Rehabilitation Engineering, vol. 25, no. 2, pp. 142-150, 2017.

[8] I. Hussain, G. Spagnoletti, G. Salvietti, and D. Prattichizzo, "Toward wearable supernumerary robotic fingers to compensate missing grasping abilities in hemiparetic upper limb," The International Journal of Robotics Research, vol. 36, pp. 1414-1436, 2017.

[9] I. Hussain, G. Salvietti, G. Spagnoletti, and D. Prattichizzo, "The Soft-SixthFinger: a Wearable EMG Controlled Robotic Extra-Finger for Grasp Compensation in Chronic Stroke Patients," IEEE Robotics and Automation Letters, vol. 1, no. 2, pp. 1000-1006, 2016.

[10] C. Cipriani, F. Zaccone, S. Micera, and M. C. Carrozza, "On the shared control of an emg-controlled prosthetic hand: analysis of userprosthesis interaction," IEEE Transactions on Robotics, vol. 24, no. 1, pp. 170-184, 2008.

[11] E. Battaglia, J. P. Clark, M. Bianchi, M. G. Catalano, A. Bicchi, and M. K. O'Malley, "The rice haptic rocker: skin stretch haptic feedback with the pisa/iit softhand," in World Haptics Conference (WHC), 2017 IEEE, pp. 7-12, IEEE, 2017.

[12] J. D. Brown, A. Paek, M. Syed, M. K. O’Malley, P. A. Shewokis, J. L. Contreras-Vidal, A. J. Davis, and R. B. Gillespie, "Understanding the role of haptic feedback in a teleoperated/prosthetic grasp and lift task," in World Haptics Conference (WHC), 2013, pp. 271-276, IEEE, 2013.

[13] C. Antfolk, M. D'Alonzo, B. Rosén, G. Lundborg, F. Sebelius, and C. Cipriani, "Sensory feedback in upper limb prosthetics," Expert review of medical devices, vol. 10, no. 1, pp. 45-54, 2013.

[14] K. Kiguchi, T. Tanaka, and T. Fukuda, "Neuro-fuzzy control of a robotic exoskeleton with emg signals," IEEE Transactions on Fuzzy Systems, vol. 12, pp. 481-490, Aug 2004.

[15] P. Heo, G. M. Gu, S.-j. Lee, K. Rhee, and J. Kim, "Current hand exoskeleton technologies for rehabilitation and assistive engineering," International Journal of Precision Engineering and Manufacturing, vol. 13, pp. 807-824, May 2012.

[16] V. A. de Jesus Oliveira, L. Nedel, A. Maciel, and L. Brayda, "Spatial discrimination of vibrotactile stimuli around the head," in 2016 IEEE Haptics Symposium (HAPTICS), pp. 1-6, April 2016.

[17] J. F. Kaiser, "On a simple algorithm to calculate the 'energy' of a signal," in International Conference on Acoustics, Speech, and Signal Processing, pp. 381-384, April 1990.

[18] D. Yang, H. Zhang, Y. Gu, and H. Liu, "Accurate EMG onset detection in pathological, weak and noisy myoelectric signals," Biomedical Signal Processing and Control, vol. 33, pp. 306-315, 2017.

[19] X. Li, P. Zhou, and A. S. Aruin, "Teager-Kaiser energy operation of surface EMG improves muscle activity onset detection," Annals of biomedical engineering, vol. 35, no. 9, pp. 1532-1538, 2007.

[20] D. Farina and R. Merletti, "Comparison of algorithms for estimation of EMG variables during voluntary isometric contractions," Journal of Electromyography and Kinesiology, vol. 10, no. 5, pp. 337-349, 2000.

[21] K. T. Özgünen, U. Çelik, and S. S. Kurdak, "Determination of an optimal threshold value for muscle activity detection in EMG analysis," Journal of sports science \& medicine, vol. 9, no. 4, pp. 620-628, 2010. 